\title{
The Relationship Between Cigarette Smoking and Biomarkers of Exposure Across Two Study Centers in Europe
}

\author{
Frazer J. Lowe*,a, Evan O. Gregg ${ }^{\mathrm{b}}$, Antonella Bassi ${ }^{\mathrm{a}}$ and Riccardo Puntoni ${ }^{\mathrm{b}}$ \\ ${ }^{a}$ British American Tobacco, GR\&D Regents Park Road, Southampton, SO15 8TL, UK \\ ${ }^{b}$ Consultant to British American Tobacco GR\&D, Southampton, UK
}

\begin{abstract}
The relationship between biomarkers of exposure to cigarette smoke in 24h urine samples collected from groups of 80 smokers ( 44 males, 36 females) and 40 never smokers (17 males, 23 females) at two centers in Europe was studied. Eight biomarkers (nicotine, cotinine, hydroxycotinine, 4-(methylnitrosamino)-1-(3-pyridyl)-1-butanol (NNAL) and all of the respective glucuronide conjugates) were measured. Subjects from the two centers were pooled and biomarker data analyzed according to the machine smoked tar yield of the brand each subject smoked and the recorded number of cigarettes smoked per day (CPD). A statistically significant relationship between CPD and all of the biomarkers analyzed was found. Smokers of less than $11 \mathrm{CPD}$ had the lowest mean $24 \mathrm{~h}$ urinary concentrations for all biomarkers measured. However, if the amount of constituent obtained from each cigarette smoked was calculated, then the amount of nicotine obtained per cigarette was highest in this group although the variation was also greatest for this group. The amount of NNK (4-(methylnitrosamino)-1-(3-pyridyl)-1 butanone, the parent molecule of NNAL) obtained per cigarette was not statistically significantly different across all groups. In conclusion, these results confirm the reliability of $24 \mathrm{~h}$ urinary total nicotine and NNAL concentrations as biomarkers of exposure to specific cigarette smoke constituents across two centers in Europe. These measurements may provide an objective alternative to CPD when grouping smokers for are studies of other endpoints.
\end{abstract}

Keywords: Biomarker, smoking, NNAL, nicotine, NNK, cotinine.

\section{INTRODUCTION}

Cigarette smoke is a complex mixture of over 5000 different chemicals that partition between a gaseous and a particulate phase [1]. Interaction between the smoke constituents adds further complexity to the mixture. The yields of smoke constituents per cigarette are measured by machine under controlled smoking conditions defined by the International Organization for Standardization [2] or the USA Federal Trade Commission [3]. While these methods are useful for measuring the machine derived yields of cigarette smoke constituents, they do not, and were not intended to, reflect human smoke yield [4]. Individual smoking behavior, such as puff volume, puff frequency and depth of inhalation, determines the amount of smoke any smoker draws into the lungs [5].

The limits of machine smoked measurements of constituent yields point to the need for other systems to measure the exposure to cigarette smoke. In other studies with large numbers of subjects, a technique based on the analysis of smoked cigarette filters has been developed [6]. This method has the advantage of giving an estimate of mouth-level exposure for the cigarette actually smoked by each smoker without the need for the collection of any biofluid; however, it does not measure the amount of smoke drawn into the lungs or subsequently distributed throughout body tissues and so

*Address correspondence to this author at the British American Tobacco, GR\&D Regents Park Road, Southampton, SO15 8TL, UK; Tel: +44 (0)2380 793647; Fax: +44 (0)2380 793076; E-mail: Frazer_Lowe@bat.com interest has focused on the potential of biomarkers for this use [7]. Biomarkers of exposure are used to confirm the absorption of specific smoke constituents in a quantitative manner, avoiding the problems of smoking behavior and machine yield measurement. Nonetheless, covering the wide range of constituents present in tobacco smoke will require the measurement of several biomarkers. Ideally, biomarkers of exposure should be related to a specific exposure of interest, such as the measurement of urinary 4-(methylnitrosamino)-1-(3-pyridyl)-1-butanol (NNAL) as a biomarker of exposure to 4-(methylnitrosamino)-1-(3-pyridyl)-1 butanone (NNK) [8].

We have previously described two separate studies conducted at geographically distinct sites across Europe: Leeds, UK [9] and Messina, Italy [10], in which the subjects were recruited according to similar criteria. Although there was a similarity between the recruitment criteria, there are distinct differences between the diets and lifestyles of these regions and the brands of cigarettes smoked were also different. In the present study, these subjects were pooled and the original urinary biomarkers of exposure data from Leeds [9] and Messina [10] were re-examined with different statistical approaches. This larger subject pool allowed us to examine sub-groups in cigarettes per day (CPD) categories that are different from the original recruiting criteria and to establish whether data obtained from two separate European areas is suitable for combination, allowing for possible lifestyle and cigarette brand differences. The results confirm the utility of biomarkers for evaluating exposure to specific cigarette smoke constituents. 


\section{MATERIALS AND METHODOLOGY}

The detailed description of the subject recruitment and study design at both centers has been described previously $[9,10]$. Briefly, never smokers, former smokers and regular smokers of cigarettes from two categories were recruited based on screening questionnaire responses and spot salivary or urinary cotinine measurements and exhaled breath carbon monoxide measurements, according to pre-defined inclusion and exclusion criteria. The two groups of cigarette smokers included were a "lower exposure" group (subjects who regularly smoked 10 or less cigarettes per day of a specified brand with an ISO tar yield of $6 \mathrm{mg}$ or less) and a "higher exposure group" (subjects who regularly smoked 20 or more cigarettes per day of a specified brand with an ISO tar level of $9 \mathrm{mg}$ or greater). All study subjects were recruited as healthy volunteers, as assessed independently by routine medical examination at the clinical centers.

For this study subjects from both centers were pooled but as only one centre recruited former smokers they have not been included in the analyses presented here. The new pooled group contained 80 smokers (44 males, 36 females) and 40 never smokers (17 males, 23 females). The mean age of the smoker subjects was 32.9 years (SD 9.7) and the never smoker subjects 29.1 years (SD 7.2). Eight biomarkers of exposure were measured in $24 \mathrm{~h}$ urine from these groups (nicotine, cotinine, hydroxycotinine, NNAL and all of the respective glucuronide conjugates). NNAL and its metabolite were analyzed using a solid phase extraction and liquid

Table 1. Exposure Biomarkers by Cigarette Yield and Number of Cigarettes Smoked Per Day

\begin{tabular}{|c|c|c|c|c|c|c|}
\hline & & $\mathbf{N}$ & Median & Geometric Mean & Confidence & $95 \%$ \\
\hline \multirow{6}{*}{$\begin{array}{l}\text { Hydroxycotinine/ } \\
\text { Creatinine* } \\
(\mu \mathrm{g} / \mathrm{mg})\end{array}$} & NS & 40 & 3.54 & 9.20 & 5.59 & 15.09 \\
\hline & $1-10 \mathrm{LT}$ & 27 & 2659.72 & 2440.22 & 1796.40 & 3314.78 \\
\hline & $11-18 \mathrm{LT}$ & 13 & 3002.71 & 2325.00 & 1338.98 & 4037.12 \\
\hline & $11-18$ & 13 & 4269.79 & 4373.14 & 3046.88 & 6276.72 \\
\hline & $19-24$ & 13 & 8150.94 & 7058.31 & 4858.05 & 10255.22 \\
\hline & $25+$ & 14 & 7522.16 & 8938.55 & 6646.63 & 12020.77 \\
\hline \multirow{6}{*}{$\begin{array}{l}\text { Nicotine/Creatinine* } \\
(\mu \mathrm{g} / \mathrm{mg})\end{array}$} & NS & 40 & 0.49 & 2.23 & 1.79 & 2.79 \\
\hline & 1-10LT & 27 & 556.75 & 475.82 & 345.38 & 655.51 \\
\hline & $11-18 \mathrm{LT}$ & 13 & 1232.80 & 936.21 & 527.91 & 1660.29 \\
\hline & $11-18$ & 13 & 1113.65 & 1193.97 & 875.31 & 1628.64 \\
\hline & $19-24$ & 13 & 2016.04 & 1935.16 & 1406.20 & 2663.03 \\
\hline & $25+$ & 14 & 2123.99 & 1976.66 & 1442.20 & 2709.19 \\
\hline \multirow{6}{*}{$\begin{array}{l}\text { Cotinine/Creatinine* } \\
(\mu \mathrm{g} / \mathrm{mg})\end{array}$} & NS & 40 & 1.98 & 5.05 & 3.15 & 8.07 \\
\hline & 1-10LT & 27 & 1555.31 & 1271.59 & 881.09 & 1835.15 \\
\hline & $11-18 \mathrm{LT}$ & 13 & 2080.42 & 2040.05 & 1261.35 & 3299.47 \\
\hline & 11-18Cig & 13 & 2584.87 & 2789.17 & 2090.18 & 3721.93 \\
\hline & $19-24$ & 13 & 4839.87 & 4305.2 & 3555.08 & 5213.73 \\
\hline & $25+$ & 14 & 4328.48 & 5343.08 & 4177.12 & 6834.50 \\
\hline \multirow{6}{*}{$\begin{array}{l}\text { Total Nicotine Metabol./ } \\
\text { Creatinine* }(\mu \mathrm{g} / \mathrm{mg})\end{array}$} & NS & 40 & 0 & 123.36 & 85.60 & 177.79 \\
\hline & $1-10 \mathrm{LT}$ & 27 & 5152.90 & 5352.47 & 3639.00 & 7872.77 \\
\hline & $11-18 \mathrm{LT}$ & 13 & 5639.90 & 4838.85 & 2906.73 & 8055.28 \\
\hline & $11-18$ & 13 & 7360.90 & 8034.90 & 5915.96 & 10912.79 \\
\hline & $19-24$ & 13 & 14903.02 & 14141.48 & 9957.81 & 20082.86 \\
\hline & $25+$ & 14 & 20173.01 & 23759.74 & 16708.55 & 33786.62 \\
\hline \multirow{6}{*}{$\begin{array}{l}\text { NNAL/Creatinine* } \\
\text { (pg.mg) }\end{array}$} & NS & 40 & 5.00 & 4.72 & 3.78 & 5.89 \\
\hline & 1-10LT & 27 & 90.13 & 69.55 & 49.44 & 97.83 \\
\hline & $11-18 \mathrm{LT}$ & 13 & 129.20 & 114.40 & 69.73 & 187.68 \\
\hline & $11-18$ & 13 & 198.67 & 162.52 & 112.51 & 234.75 \\
\hline & $19-24$ & 13 & 220.11 & 200.75 & 150.97 & 266.96 \\
\hline & $25+$ & 14 & 270.86 & 236.96 & 172.84 & 324.87 \\
\hline
\end{tabular}

$\mathrm{NS}=$ never smoker $\mathrm{LT}=$ lower tar cigarettes with a tar yield $<6 \mathrm{mg}$ based on the International Organization for Standards (ISO) machine smoking yield. * P for trend: $<0.01$. 
chromatography with tandem mass spectrometry (LC/MS/MS) method based upon methods by Xia et al. [11] and Pan et al. [12] (LLOQ 5ng/ml ULOQ 1000ng/ml). Urinary nicotine and its metabolites were analyzed using a solid phase extraction and liquid chromatography with tandem mass spectrometry as described by St. Charles et al. [6] (LLOQ 10ng/ml ULOQ 5000ng/ml). When described below, total nicotine metabolites represent the sum of the nicotine, cotinine and hydroxycotinine plus all of their glucuronide conjugates, allowing for the molecular mass of the analyte, expressed as a mass of the parent molecule. The glucuronide moiety of NNAL is deconjugated enzymatically prior to analysis and therefore the sum of both glucuronide conjugated and unconjugated NNAL is presented.

Although recruited according to self-declared smoking amounts, during the $24 \mathrm{~h}$ clinical observation period, many smokers smoked a different number of CPD than they had reported in the screening interview, with 26 subjects smoking between 11 - 18 CPD. Therefore, all subjects were divided into new sub-groups according to the number of CPD recorded in the clinical period and the ISO machine smoked tar yield of their cigarette brands as follows:

Never smokers $(\mathrm{n}=40) ; 1-10$ CPD, <6mg tar yield $(\mathrm{n}=27)$; 11-18 CPD, <6mg tar yield $(\mathrm{n}=13) ; 11-18$ CPD,$\geq$ $6 \mathrm{mg}$ tar yield $(\mathrm{n}=13) ; 19-24 \mathrm{CPD}, \geq 6 \mathrm{mg}$ tar yield $(\mathrm{n}=13)$; and $\geq 25 \mathrm{CPD}, \geq 6 \mathrm{mg}$ tar yield $(\mathrm{n}=14)$.

\section{Statistical Analysis}

The median value, geometric mean and their confidence limits were calculated for all biomarker concentrations. A chi square test for trend and analysis of variance across groups were performed. In all trend analyses, the never smoker group data was included as the baseline. Although outlier and extreme data points are shown separately in the figures presented, all data have been used in the statistical analyses.

\section{Clinical Ethics}

These studies were conducted in accordance with Good Clinical Practice, the Declaration of Helsinki (as adopted by the 18th World Medical Assembly in 1964 and reviewed in Scotland in 2000), and the European Legislation on Clinical Trials. The protocols for each of the clinical studies were approved by the Local Independent Ethics Committees: The Messina protocol by the University Polyclinic "G Martino" (Messina, Italy) with the registration number E468/06 and the Leeds protocol by the Covance Clinical Research Unit Independent Ethics Committee. Written, informed consent was obtained from all subjects prior to the start of the study.

\section{RESULTS}

A statistically significant positive trend with the number of CPD was observed for all the exposure biomarkers studied (Table 1).

Due to the sub-group split of the subjects it was possible to directly compare biomarkers of exposure in smokers of $11-$ 18 CPD for cigarettes with an ISO tar yield < $6 \mathrm{mg}(\mathrm{n}=13)$ and those with an ISO tar yield of $\geq 6 \mathrm{mg}(\mathrm{n}=13)$. For all of the biomarkers of exposure examined, lower concentrations were seen in smokers of lower tar yield cigarettes compared to higher tar yield cigarettes, although the differences did not reach statistical significance. Analysis of heterogeneity did not show a "center effect" for any of the biomarkers of exposure although there was some effect in the number of CPD, with the subjects from Leeds having greater proportion of more CPD smokers (data not shown).

In Fig. (1), the relationship between total nicotine metabolites or total NNAL and the number of CPD is shown.

For both biomarkers there is a clear linear trend across the groups from never smokers to smokers of $\geq 25 \mathrm{CPD}$. From these data the average contribution of each cigarette smoked were calculated by dividing the concentration of the biomarkers by the CPD, which is shown in Fig. (2).

From Fig. (2), it can be seen that smokers of less than 11 CPD had the highest median nicotine metabolite concentration per cigarette, but this group also had the highest IQR of nicotine per cigarette, using this simple calculation approach. Even allowing for this wide variation, there is not a clear-cut

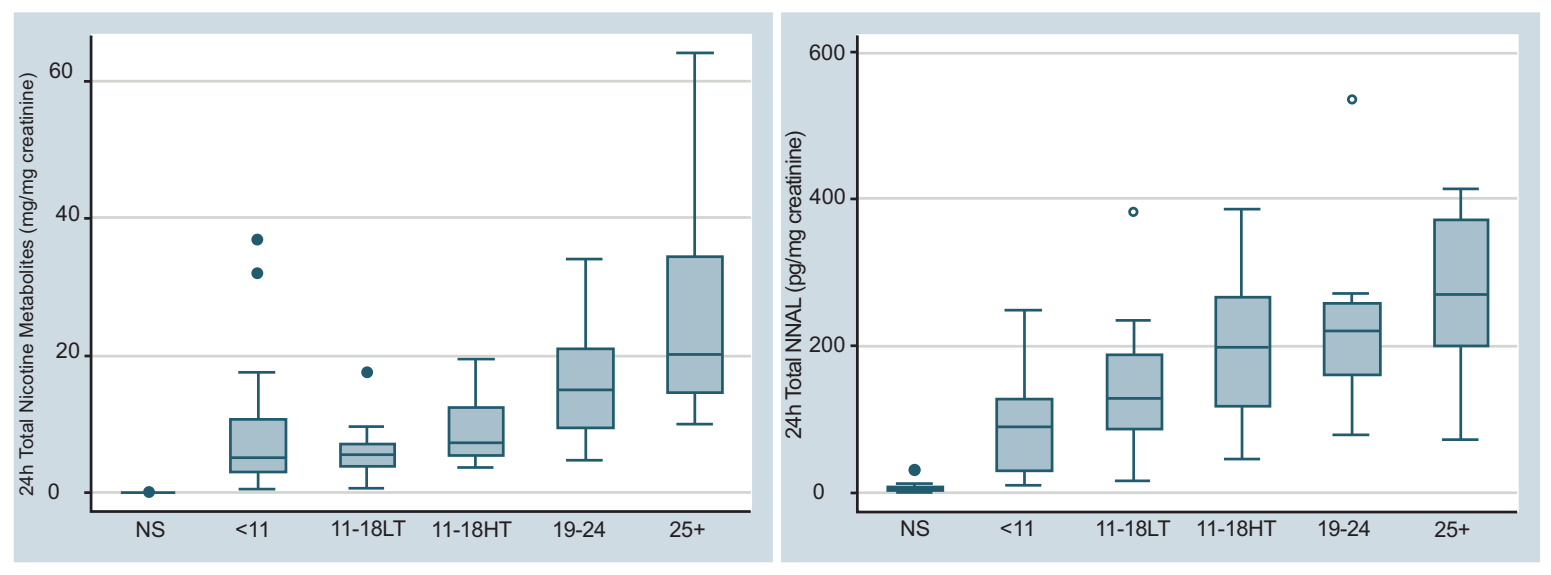

Fig. (1). Box plots for $24 \mathrm{~h}$ urinary total nicotine metabolites (left panel) and total NNAL (right panel) with subjects grouped by CPD and cigarette type. The sample median (solid line) and interquartile range (IQR) (shaded area) are shown. Whisker bars connect the highest and lowest data points within the upper quartile plus $1.5 \mathrm{IQR}$ and the lower quartile minus 1.5 IQR. Outliers are values between $1.5 \mathrm{IQRs}$ and 3 IQRs from the end of a box and are shown as open circles. Values more than $3 \mathrm{IQR}$ 's from the end of a box are defined as extreme (solid circles).

NS = never smokers, $\mathrm{LT}=$ lower $\operatorname{tar}(<6 \mathrm{mg})$ and $\mathrm{HT}=$ higher $\operatorname{tar}(\geq 6 \mathrm{mg})$. 

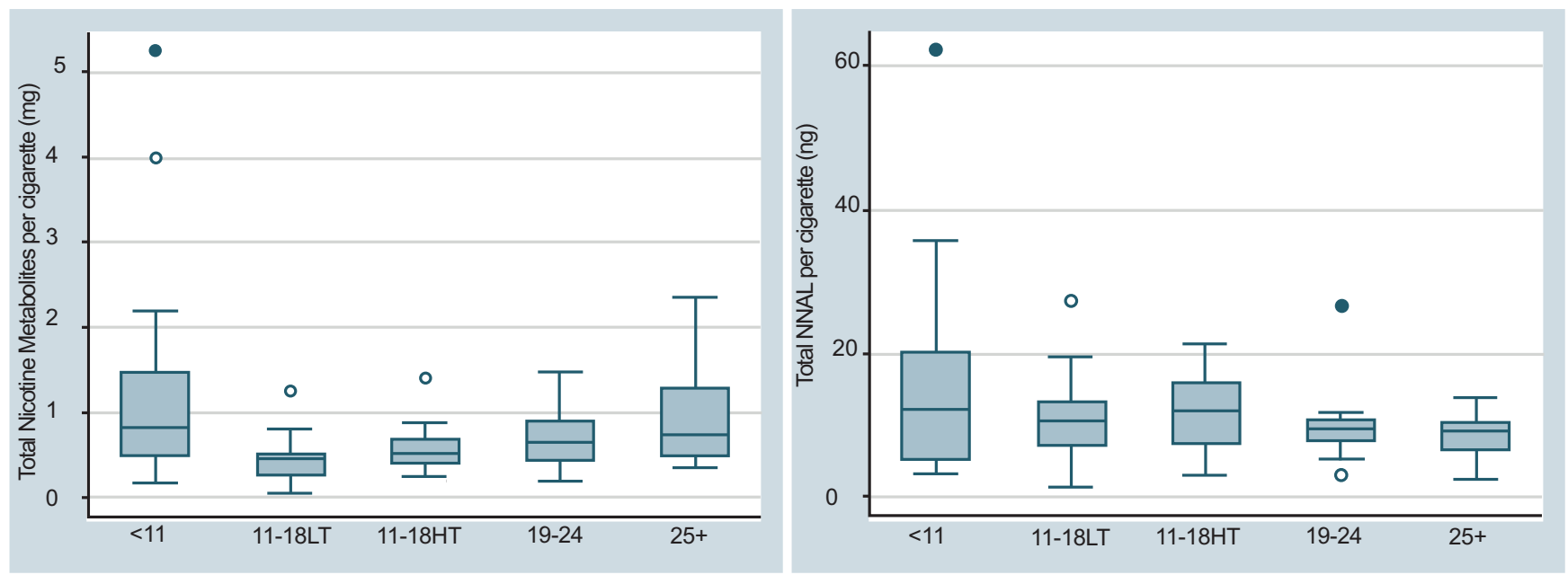

Fig. (2). Box plots for total nicotine metabolites per cigarette (left panel) and total NNAL per cigarette (right panel) with subjects grouped by CPD and cigarette type. The sample median (solid line) and interquartile range (IQR) (shaded area) are shown. Whisker bars connect the highest and lowest data points within the upper quartile plus 1.5 IQR and the lower quartile minus 1.5 IQR. Outliers are values between 1.5 IQRs and 3 IQRs from the end of a box and are shown as open circles. Values more than 3 IQR's from the end of a box are defined as extreme (solid circles).

LT $=$ lower $\operatorname{tar}(<6 \mathrm{mg})$ and HT $=$ higher $\operatorname{tar}(\geq 6 \mathrm{mg})$.

negative association between CPD and total nicotine metabolites. Indeed, without the $<11$ CPD group, no clear association between the relationship between urinary nicotine metabolites per cigarette and CPD is obvious. For NNAL, a urinary metabolite of NNK, there is no apparent positive or negative trend for NNK obtained per cigarette smoked across the sub-groups.

\section{DISCUSSION}

The biomarkers of exposure to cigarette smoke constituents that were measured indicate the amount of nicotine, total nicotine metabolites and total NNAL present in $24 \mathrm{~h}$ urine samples of smokers and, therefore, provide objective information on individual exposure. In this study, the information has been supplemented with data obtained from detailed questionnaires and recorded smoking behavior both outside and within a clinical environment. The presence of a clear and positive relationship between all exposure categories - the recruitment groups, observed CPD and biomarkers of exposure - may prove to be useful in evaluation of health risks associated with smoking and in the evaluation of potential reduced-exposure products [7, 13].

The results of this combined analysis show that:

- $\quad$ increasing the number of cigarettes smoked increases the levels of smoke constituents and their metabolites found in the urine, with a clear and statistically significant positive relationship (Table 1);

- smokers of cigarettes with a machine smoked tar yield $<6 \mathrm{mg}$ (ISO) have lower concentrations of the biomarkers of exposure of interest in this study compared to subjects smoking the same number of CPD with a machine smoking tar yield $\geq 6 \mathrm{mg}$ (Table 1 and Fig. 1); and

- $\quad$ smokers of less than 11 CPD have the lowest median concentrations of nicotine metabolites and NNAL in their urine samples (Table $\mathbf{1}$ and Fig. 1) but they also obtain higher median amounts of nicotine from each cigarette (Fig. 2). This extreme variation may be due to the known wide variation in puffing profiles for smokers of cigarettes from this category [14].

It was expected that a clear "center effect" would be seen in the urinary NNAL concentrations because of the well documented differences in nitrosamine levels in tobacco blends used in Virginia cigarettes (favored by smokers in the UK) and blended cigarettes (favored by smokers in Europe) [15]. This was not seen in the pooled data, possibly because approximately half of the smokers from the Leeds arm smoked blended products and the total numbers for any single brand in the study were low. Even if the Leeds data were considered separately, statistically significant heterogeneity in the urinary NNAL concentration across brands was not observed (data not shown). However, without data to confirm machine smoked NNK deliveries from each of the cigarette brands used in the studies, it is difficult to assess whether or not any center differences should be expected.

Although the health risks of smoking are clear [16], the relationship between specific tobacco smoke constituents and mechanistic steps in diseases remains unclear [17-19]. Epidemiological studies linking biological markers of exposure with biomarkers of effect and risk or potential harm are needed to be able to better understand the development of diseases associated with smoking. Furthermore, the biomarkers of exposure to cigarette smoke currently available are all markers of relatively short-term exposure. Nicotine and its metabolites are suitable as biomarkers for recent exposure within 1 to 3 days [20] and NNAL gives an indication of exposure to NNK over a period of weeks to months [21]. This may lead to a possibility of artifact in any analysis like the one presented in Fig. (2) because short-term influences on smoking behavior (e.g. CPD), such as the attendance at a clinical center, will have a disproportionate impact on urinary nicotine metabolite compared to NNAL concentrations. 
Epidemiological studies would benefit from the availability of biomarkers that reflect cigarette smoke exposure over a period of months to several years. When all of these baseline data are firmly established, the scientific community will be better placed to evaluate potential reduced-exposure products, as suggested by other groups [7, 13].

\section{CONCLUSION}

These results confirm the reliability of biomarkers of exposure for evaluation of short-term exposure to cigarette smoke constituents, without the need to take into account differences in individual smoking behavior and, for nicotine, metabolism. The pooled statistical analysis used in this study suggests that these biomarkers are suitable for use across distinct European geographical recruitment areas.

\section{CONFLICT OF INTEREST STATEMENT}

This study was funded by British American Tobacco. The authors declare no conflict of interest outside employment and funding source.

\section{REFERENCES}

[1] Rodgman A, Perfetti T. The Chemical Components of Tobacco and Tobacco Smoke. Winston-Salem USA : CRC Press 2009.

[2] International Organisation for Standardisation. ISO 3308. Routine analytical smoking machine - definition and standard conditions. Geneva 2000.

[3] Federal Trade Commission. Cigarettes. Testing for tar and nicotine content - statements for consideration. Fed Reg 1967; 32: 11178.

[4] Baker RR. The development and significance of standards for smoking machine methodology. Beitr Tabakforsch Intl 2002; 20: $23-41$.

[5] Baker RR, Dixon M. The retention of tobacco smoke constituents in the human respiratory tract. Inhal Toxicol 2006; 18: 255-94.

[6] St Charles FK, Krautter GR, Dixon M, Mariner DC. A comparison of nicotine dose estimates in smokers between filter analysis, salivary cotinine, and urinary excretion of nicotine metabolites. Psychopharmacology 2006; 189(3): 345-54.

[7] Hatsukami DK, Benowitz NL, Rennard SI, Oncken C, Hecht SS. Biomarkers to assess the utility of potential reduced exposure tobacco products. Nicotine Tobacco Res 2006; 8(2): 169-91.
Hecht SS, Carmella SG, Chen M, et al. Quantitation of urinary metabolites of a tobacco-specific lung carcinogen after smoking cessation. Cancer Res 1999; 59(3): 590-6.

[9] Lowe FJ, Gregg EO, McEwan M. Evaluation of biomarkers of exposure and potential harm in smokers, former smokers and never-smokers. Clin Chem Lab Med 2009; 47(3): 311-20

[10] Calapai G, Caputi AP, Mannucci C, et al. Cardiovascular biomarkers in groups of established smokers after a decade of smoking. Basic Clin Pharmacol Toxicol 2009; 104(4): 322-8.

[11] Xia Y, McGuffey JE, Bhattacharyya S, et al. Analysis of the tobacco-specific nitrosamine 4-(methylnitrosamino)-1-(3-pyridyl)-1butanol in urine by extraction on a molecularly imprinted polymer column and liquid chromatography/atmospheric pressure ionization tandem mass spectrometry. Anal Chem 2005 1; 77(23): 7639-45.

[12] Pan J, Song Q, Shi H, et al. Development, validation and transfer of a hydrophilic interaction liquid chromatography/tandem mass spectrometric method for the analysis of the tobacco-specific nitrosamine metabolite NNAL in human plasma at low picogram per milliliter concentrations. Rapid Commun Mass Spectrom 2004; 18(21): 2549-57.

[13] Stratton K, Shetty P, Wallace R, Bondurant S. Eds. Clearing the smoke. Assessing the science base for tobacco harm reduction. Board of Health Promotion, Institute of Medicine. Washington DC: National Academy Press 2001.

[14] Scherer G. Smoking behaviour and compensation: a review of the literature. Psychopharmacology 1999; 145(1): 1-20.

[15] Davis MT, Nelson DL. Eds. Tobacco production, chemistry and technology. Oxford: Blackwell Science 1999.

[16] World Health Organisation. World Health Report 2002: Reducing risks, promoting healthy life. World Health Organisation: Geneva 2002.

[17] Yanbaeva DG, Dentener MA, Creutzberg EC, Wesseling G, Wouters EMF. Systemic effects of smoking. Chest 2007; 131: 1557-66.

[18] World Health Organisation TobReg study group. The scientific basis of tobacco product regulation. World Health Organisation Technical Report Series 945: Geneva 2007.

[19] Hatsukami DK, Hanson K, Briggs A, et al. Clinical trials methods for evaluation of potential reduced exposure products. Cancer Epidemiol Biom Prev 2009; 18(12): 3143-95.

[20] Tricker AR. Biomarkers derived from nicotine and its metabolites: A review. Beitr Tabakforsch Intl 2006; 22: 147-75.

[21] Hecht SS, Murphy SE, Carmella SG, et al. Effects of reduced cigarette smoking on the uptake of a tobacco-specific lung carcinogen. J Natl Cancer Inst 2004; 96: 107-15. 